\title{
Editorial:
}

\section{Towards a Better Understanding of Aggression and Other Related Concepts}

This special issue entitled "Towards a better understanding of aggression and other related concepts" is a product of the XXXVII CICA International Conference co-organized by two Polish universities: Kazimierz Wielki University of Bydgoszcz and the University of Zielona Góra. It took place from the 22 to the 25 June, 2014 and was attended by about 100 participants from 16 countries [1].

The aim of the Conference was to study the phenomena of aggression and conflict resolution using a comprehensive, integrated and interdisciplinary approach which takes into account both biological and psycho-socio-cultural factors. Several communications dealing with emotions, including anger and fear, and others with behaviors such as aggression, violence and terrorism, have been selected for this issue.

A Southafrican practitioner, Tina Lindhard, specialized in transpersonal psychology, suggests that maybe it is time we start studying emotions including anger and fear from "the inside out" by including phenomenology as a method to throw more light on how we experience these states in or through our bodies. Furthermore, she presents the "Living Matrix" model, which owes its origin to Quantum Mechanics and Electronic Biology, as a new complementary way of understanding how the living organism functions [2].

The Italian scholar Dr. Pagani stresses the complexity of violence, presented as a macrosystem of networks and of agents linked and interacting at different interconnected levels. She points out to the difficulty of defining violence, referring it not only to the explorations of the connections between systems taken from different research fields, but also to the theoretical premises and to the aims of the research. She argues that this "holistic" approach could allow a deeper understanding of violence and could lead towards more innovative and effective solutions to the problem of violence itself [3].

Dr. Ramirez, who has dedicated several decades of his research to the analysis of the justification of aggression from a cross-cultural approach across four continents, evaluates the applicability of a specific test (CAMA) in a new cultural context, assessing the structural equivalence of the data obtained in two different German age cohorts with the data previously investigated across the other cultures. Some adaptations concerning the assessment and theoretical models of the justification of aggressive actions in the German cultural context are being discussed [4].

Two academic colleagues from the University of Zielona Góra, Dr. Farnicka \& Dr. Grzegorzewska, focus on some more practical aspects of aggression research, if we may say that, leading towards its prevention or therapy in children and adolescents. These Polish psychologists identify and analyse the family determinants for undertaking the aggressor or victim role. The results of their study reveal a number of determinants for people involved in perpetration or victimization, such as the type of relationship with parents (secure or insecure pattern), personal experience of being in the victim or aggressor role, and the level of hostility [5].

Finally, the first president of the Society for Terrorism Research, Dr. LoCicero, recounts some concerns raised by American psychologists, both earlier, in the years following September 11, 2001 (9/11), and more recent changes in the US policy, leading towards the risk for the USA of becoming a police state. According to her paper, engaging in open discussion about the failings of the American policy, the sometimes legitimate grievances of terrorist groups, and the draw of violence as a solution, is likely to put sincere and innocent adults at risk of becoming targets of intensive surveillance and suspicion [6].

It is thus clear that the discussion on aggression and other related concepts is here carried out from various scientific perspectives, which include traditional experimental psychology with a special focus on the role of family relationships and cultural factors, social and political psychology with a special focus on the role of State policies, and other theoretical perspectives which try to integrate their psychological framework with contributions from western and eastern philosophy, the neurosciences, biology, quantum physics, and complexity theory.

\section{REFERENCES}

[1] Pagani C, Farnicka M, Liberska H, Ramirez JM, Eds. Conflict and aggression: developmental and social conditions. DIFIN: Warsaw 2014.

[2] Lindhard T, Emotions including anger, fear, body sensations and inner experiencing. Open Psychol J 2015; 8: 3-10

[3] Pagani C. Violence and complexity. Open Psychol J 2015; 8: 11-16.

[4] Ramirez JM. Structural equivalence of the questionnaire on moral attitudes toward aggression (CAMA) in different German age groups. Open Psychol J 2015; 8: 17-22. 
[5] Farnicka M, Grzegorzewska I. The role of social support in determining the acting as a victim or aggressor during different periods of adolescence. Open Psychol J 2015; 8: 23-31.

[6] LoCicero A. Unintended domestic consequences of America's "War on Terrorism". Open Psychol J 2015; 8: 32-37.

\section{J. Martin Ramirez}

Center for Conflict Studies, Nebrija University,

Madrid

\section{Camilla Pagani}

Institute of Cognitive Sciences and Technologies

National Research Council, Rome 